\title{
Treatment of radiation-induced acute intestinal injury with bone marrow-derived mesenchymal stem cells
}

\author{
KAI ZHENG ${ }^{1}$, WEIZHEN WU ${ }^{1}$, SHUNLIANG YANG ${ }^{1}$, LIANGHU HUANG ${ }^{1}$, JIN CHEN $^{1}$, \\ CHUNGUI GONG ${ }^{2}$, ZHICHAO FU ${ }^{2}$, RUOFEI LIN ${ }^{1}$ and JIANMING TAN ${ }^{1}$ \\ ${ }^{1}$ Cell and Organ Transplant Institute; ${ }^{2}$ Radiotherapy Centre, Fuzhou General Hospital, Fuzhou, Fujian 350025, P.R. China
}

Received October 16, 2014; Accepted October 21, 2015

DOI: $10.3892 / \mathrm{etm} .2016 .3248$

\begin{abstract}
The aim of the present study was to investigate the ability of bone marrow-derived mesenchymal stem cells (BMSCs) to repair radiation-induced acute intestinal injury, and to elucidate the underlying repair mechanism. Male Sprague-Dawley rats were subjected to whole abdominal irradiation using a single medical linear accelerator (12 Gy) and randomly assigned to two groups. Rats in the BMSC-treated group were injected with $1 \mathrm{mlBMSC}$ suspension $\left(2 \times 10^{6} \mathrm{cells} / \mathrm{ml}\right)$ via the tail vein, while the control group rats were injected with normal saline. BMSCs were identified by detecting the expression of CD29, CD90, CD34 and CD45 using flow cytometry. The expression of the cytokines stromal cell-derived factor 1 (SDF-1), prostaglandin E2 (PGE2) and interleukin (IL)-2 was detected using immunohistochemical techniques. Plasma citrulline concentrations were evaluated using an ELISA kit. Rat general conditions, including body weight, and changes in cellular morphology were also recorded. The results suggested that BMSCs exerted a protective effect on radiation-induced acute intestinal injury in rats. The histological damage was rapidly repaired in the BMSC-treated group. In addition, the BMSC-treated group showed significantly reduced radiation injury scores $(\mathrm{P}<0.01)$, mildly reduced body weight and plasma citrulline levels, significantly more rapid recovery $(\mathrm{P}<0.01)$, significantly reduced expression of the cytokines PGE2 and IL-2 $(\mathrm{P}<0.05)$ and significantly increased SDF-1 expression $(\mathrm{P}<0.01)$ compared with the control group. In summary, the present results indicate that BMSCs are able to effectively reduce inflammation and promote repair of the structure and function of intestinal tissues damaged by radiation exposure, suggesting that they may provide a promising therapeutic agent.
\end{abstract}

Correspondence to: Professor Jianming Tan, Cell and Organ Transplant Institute, Fuzhou General Hospital, 56 Xierhuan North Road, Fuzhou, Fujian 350025, P.R. China

E-mail: jianmingtancn@126.com

Key words: cell therapy, bone marrow mesenchymal stem cells, radiation intestinal injury, rats

\section{Introduction}

Radiation damage may occur by exposure to various types of technology which employ nuclear energy, including devices used in power generation, geological exploration, industrial inspection, medical exposure and radiation sterilization (1). The limitations of radiotherapy, in particular radiation injury to the gastrointestinal tract, have produced an urgent requirement for novel and effective treatments for radiation damage (2). Previous studies have shown that mesenchymal stem cells (MSCs) are able to promote the repair of intestinal structures and functions, indicating their potential for the treatment of intestinal radiation injury (3-5).

Francois et al (6) reported the transplantation of bone marrow-derived MSCs (BMSCs) into intestinal tissues subjected to radiation injury. Furthermore, Okamoto et al (7) detected donor-derived epithelial cells in the intestinal epithelium of BMSC-transplanted recipient rats, confirming that BMSCs are able to differentiate into intestinal epithelial cells. Linard et al (3) demonstrated that BMSCs are able to proliferate in the intestinal tract and promote the repair of the intestinal tissues damaged by radiation. Another study reported that, although MSCs have been observed in the gut, the intestinal transplantation rate was low (8). MSC-induced repair has been reported in intestinal tract tissues following radiation-induced damage (9); however, the optimum cell type, dose, treatment course and the mechanisms underlying MSC-mediated damage repair remain unclear (10).

In the present study, a rat model of radiation-induced acute intestinal injury was established using linear accelerators in order to investigate the ability of BMSCs to repair radiation-induced acute intestinal damage. In addition, the potential repair mechanisms involved were preliminarily studied by monitoring the expression of a number of cytokines, including interleukin (IL)-2, prostaglandin E2 (PGE2) and stromal cell-derived factor 1 (SDF-1).

\section{Materials and methods}

Isolation and culturing of BMSCs. A total of 40 male Sprague-Dawley (SD) rats (age, 4-6 weeks) were provided by the Shanghai SLAC Laboratory Animal Co., Ltd. (Shanghai, China), and were sacrificed by neck dislocation, while anesthetized with $2 \%$ pentobarbital sodium (Sigma-Aldrich, St. Louis, 
MO, USA). The femur and tibia were separated under sterile conditions to expose the bone marrow cavity, which was rinsed with saline. The bone marrow filtrate was collected and centrifuged at $225 \mathrm{x}$ g for $5 \mathrm{~min}$. The supernatant was discarded and the cells were resuspended in HyClone low-glucose (LG)-Dulbecco's modified Eagle's medium (DMEM; GE Healthcare Life Sciences, Logan, UT, USA) at $1 \times 10^{6}$ cells per $100 \mu \mathrm{l}$. The cell suspension was gradually added to a rat lymphocyte separation medium (Sigma-Aldrich) at a ratio of $1: 1$ and centrifuged at $978 \mathrm{x}$ g for $20 \mathrm{~min}$. A milky turbid mononuclear cell layer (the separation between the supernatant liquid) was collected and the cells were resuspended in LG-DMEM medium without fetal bovine serum (FBS) at $1 \times 10^{6}$ cells per $100 \mu \mathrm{l}$, then centrifuged at $225 \mathrm{x}$ g for $5 \mathrm{~min}$ and the pelleted cells were collected. The cells were resuspended in LG-DMEM complete medium containing $10 \% \mathrm{FBS}$ in $5 \% \mathrm{CO}_{2}$ saturated humidity at $37^{\circ} \mathrm{C}$. The culture medium was changed every 3 days, and was subcultured at a ratio of $1: 3$ when the cell confluence reached $80-90 \%$. This study was conducted in strict accordance with the recommendations in the Guide for the Care and Use of Laboratory Animals of the National Institutes of Health (1996, 7th ed.). The animal use protocol has been reviewed and approved by the Institutional Animal Care and Use Committee of Fuzhou General Hospital (Fuzhou, China). Written informed consent was obtained from all participants.

Detection of surface antigen molecular expression. Third passage rat BMSCs showing good growth were rinsed twice with phosphate-buffered saline (PBS) and digested with $0.25 \mathrm{~g} / 1$ trypsin containing ethylenediaminetetraacetic acid (HyClone; GE Healthcare Life Sciences). The cell suspension was collected and centrifuged at $225 \mathrm{x} \mathrm{g}$ for $5 \mathrm{~min}$. The pelleted cells were resuspended in PBS to achieve $1 \times 10^{6}$ cell density. The cells were incubated with phycoerythrin (PE) or fluorescein isothiocyanate (FITC)-labeled mouse anti-rat CD34 (1:200; 11-0341), CD45 (1:100; 11-0451), CD29 (1:200; 12-0291) and CD90 (1:200; 17-0900) monoclonal antibodies (eBioscience, Inc., San Diego, CA, USA) at $37^{\circ} \mathrm{C}$ in the dark for $30 \mathrm{~min}$ and tested using an EPICS XL flow cytometer (Beckman Coulter, Inc., Brea, CA, USA).

Induced differentiation. Third passage rat BMSCs showing good growth were seeded in a coverslipped preset 6 -well plate with $1 \times 10^{5}$ cells/well and cultured in a $5 \% \mathrm{CO}_{2}$ humidified incubator at $37^{\circ} \mathrm{C}$. When the cell infusion rate reached $90 \%$, the following osteogenic agents were added: LG-DMEM, $10 \%$ FBS (HyClone; GE Healthcare Life Sciences), $10^{-7} \mathrm{~mol}$ dexamethasone, $10 \mathrm{mmol} \beta$-glycerophosphate, $50 \mu \mathrm{mol} / \mathrm{l}$ vitamin C (Sigma-Aldrich), $100 \mathrm{U} / \mathrm{ml}$ sodium penicillin and $100 \mu \mathrm{g} / \mathrm{ml}$ streptomycin (pH 7.4; CSPC Pharmaceutical Group Ltd., Shijiazhuang, China). The medium was changed every 3 days, and after 3 weeks of incubation Von Kossa staining was performed to detect calcified nodules. The coverslip was fixed with $4 \%$ paraformaldehyde (Sigma-Aldrich) at $37^{\circ} \mathrm{C}$ for $30 \mathrm{~min}$ after being washed three times with PBS. Then $2 \%$ $\mathrm{AgNO}_{3}$ (Sigma-Aldrich) was added in the dark for $30 \mathrm{~min}$. After washing three times with distilled water, the coverslip was placed in UV light for $1 \mathrm{~h}$ and stained with hematoxylin (Sigma-Aldrich). Then the coverslip was detected using a phase contrast microscope (CKX41; Olympus Corporation, Tokyo, Japan).

Third passage rat BMSCs showing good growth were seeded in a coverslip preset 6 -well plate seeded at $1 \times 10^{5}$ cells/well and cultured in $5 \% \mathrm{CO}_{2}$ humidified incubator at $37^{\circ} \mathrm{C}$. When the cell infusion rate reached $90 \%$, the following adipogenic induction agents were added: LG-DMEM, $10 \% \mathrm{FBS}, 10^{-6} \mathrm{M}$ dexamethasone, $0.5 \mathrm{mmol}$ IBMX solution, $10 \mu \mathrm{g} / \mathrm{ml}$ insulin, $200 \mu \mathrm{mol} 100 \mathrm{U} / \mathrm{ml}$ indomethacin, $100 \mu \mathrm{g} / \mathrm{ml}$ chain ADM (all Sigma-Aldrich) and sodium penicillin ( $\mathrm{pH}$ 7.4; CSPC Pharmaceutical Group Ltd.). The medium was changed every 3 days, and after 9 days of incubation Oil Red $O$ staining (Xiamen Tagene Biotechnology Co., Ltd., Xiamen, China) was performed.

Preparation of model and cell therapy. Rats were anesthetized with pentobarbital sodium $(40 \mathrm{mg} / \mathrm{kg})$ by intraperitoneal injection. A WDVE-6/100 medical linear accelerator (Philips Electronics United States Ltd.) was used to perform X-ray irradiation of the whole abdominal area from the xiphoid sternum to the pubic symphysis (radiation field, $5 \times 7 \mathrm{~cm}$; examined area length, $100 \mathrm{~cm}$ ), including the head, chest and limbs. The dose rate was $427 \mathrm{cGY} / \mathrm{min}$ and the total radiation dose was $12 \mathrm{~Gy}$. A total of 40 male SD rats were randomly divided into two groups ( $n=20$ per group). The control group was infused with $1 \mathrm{ml}$ saline via the tail vein immediately after irradiation, while the BMSC-treated group was infused with $1 \mathrm{ml}$ rat BMSC suspension $\left(2 \times 10^{6}\right.$ cells $\left./ \mathrm{ml}\right)$ via the tail vein immediately following irradiation. The diet of rats (standard rodent chow provided ad libitum) was monitored, and their body weights were recorded.

Plasma citrulline content measurement. Six rats from each group were selected on days 3, 7, and 14 after irradiation and punctured in their right ventricle for the collection of anticoagulant, from which fresh plasma was obtained. The plasma citrulline content was detected using a citrulline enzyme-linked immunosorbent assay (ELISA) kit (CSB-E13414r; Cusabio Biotech Co., Ltd., Wuhan, China), according to the manufacturer's instructions.

Hematoxylin and eosin (HE) staining and radiation injury score. Six rats from each group were selected on days 3, 7 and 14 after irradiation and sacrificed. Then, the ileum tissues were collected from a $20-\mathrm{cm}$ distance to the ileocecal section, washed with precooled $0.9 \%$ saline and fixed with $10 \%$ formalin. The tissues were paraffin-embedded and sliced into $4-\mu \mathrm{m}$ sections. Routine HE staining was performed, and the changes in intestinal structure were observed by light microscopy (CX21; Olympus Corporation). The villus height and intestinal gland depth were measured with VIDS semi-automatic image analyzer (Alenia Marconi Systems, Rome, Italy). The radiation injury scoring was performed according to the score standard from related references.

Immunohistochemical analysis. SDF-1 levels were measured using an immunohistochemical detection kit (SA1055; Wuhan Boster Biological Technology Ltd., Wuhan, China) according to the manufacturer's instructions. Dewaxed and hydrated paraffin sections were treated with $50 \mu 13 \%$ hydrogen 

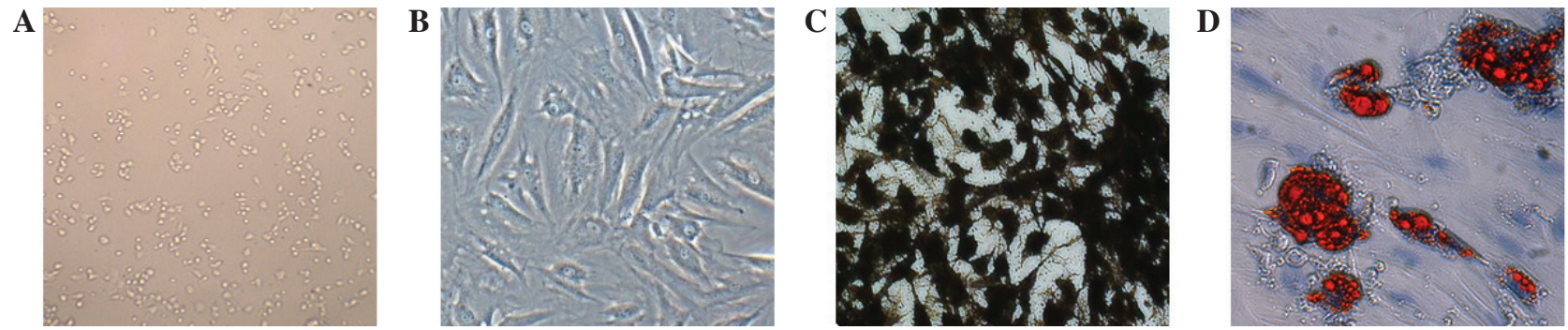

Figure 1. Morphology observation and differentiation results of rat bone marrow-derived mesenchymal stem cells (BMSCs) (magnification, x200). (A) BMSCs at generation P0 appeared as spherical cells suspended in culture medium, with marked refraction. (B) BMSCs at generation P3 appeared as fusiform cells with more uniform, rich cytoplasm, clear nucleolus, contact inhibition phenomenon occurred between the cells. (C) BMSCs osteogenic results. After Von Kossa staining, pieces of calcium nodule were observed. (D) BMSCs adipogenic results. After oil Red O staining, red fat cells were visible.
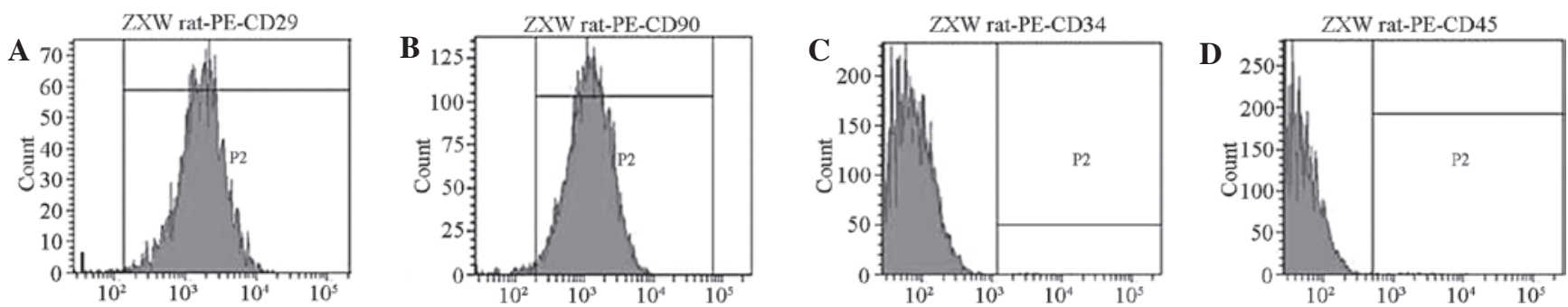

Figure 2. Surface antigen expression in rat bone marrow-derived mesenchymal stem cells (BMSCs). Flow cytometry results showed that surface antigen molecule expression of P3 generation cells. The expression absorption peaks for (A) CD29, (B) CD90, (C) CD34 and (D) CD45 were 99.25, $98.37,1.12$ and $1.03 \%$, respectively.

peroxide and incubated at room temperature for $10 \mathrm{~min}$. The sections were incubated with $50 \mu \mathrm{l}$ non-immune goat serum at room temperature for $10 \mathrm{~min}$. Next, the sections were incubated with $50 \mu \mathrm{l}$ primary rabbit anti-mouse IL-2, PGE2 or SDF-1 antibody (Wuhan Boster Biological Technology Ltd.) at room temperature for $60 \mathrm{~min}$. Finally, the sections were incubated with $50 \mu 1$ secondary goat anti-rabbit horse radish-peroxidase quick IgG-type polymer antibody at room temperature for $15 \mathrm{~min}$. The sections were then stained with 3,3'-diaminobenzidine and observed under a light microscope. Randomly, 10 fields were selected for counting under the microscope at $x 400$ magnification, and the number of positive cells was averaged.

Statistical analysis. SPSS software, version 13.0 (SPSS, Inc., Chicago, IL, USA) was used for the statistical analysis, the results of the experimental data was described using the mean \pm standard deviation and independent sample t-test. $\mathrm{P}<0.01$ was considered to indicate a statistically significant difference.

\section{Results}

Morphological observation. The observation of the primary cells from the culture flasks revealed spherical, large and small cells, with portions of cells adherent to the flask after $24 \mathrm{~h}$. The density of the adherent cells increased after 48-72 $\mathrm{h}$ culturing, and these cells were primarily fusiform, star and round shaped, with various lengths and uneven pseudopodia thickness. The number of cells increased after 3-5 days of culturing, and a large number of cell colonies showing a uniform morphology and long spindle-like arrangement were observed (Fig. 1A).

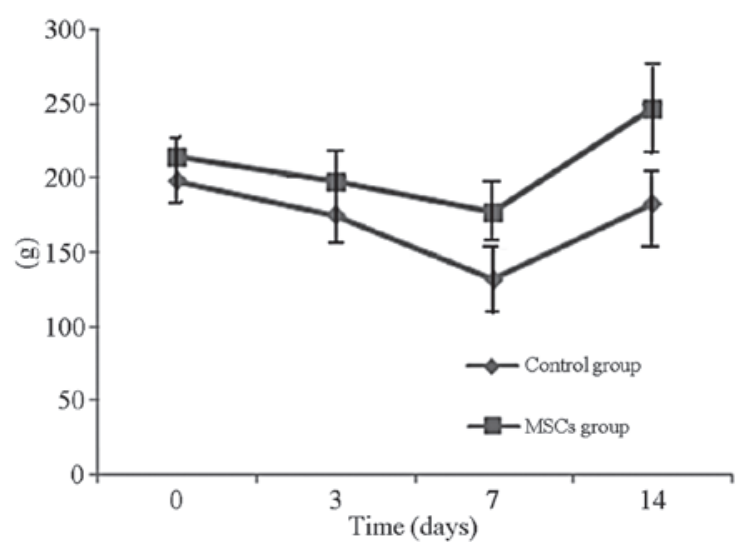

Figure 3. Differences in rat body weight following irradiation. After irradiation, the mental state of the rats was poor, food intake was reduced and thin mucus feces were excreted. The body weight of rats declined, reaching the lowest point on day 7. The rat body weight in the bone marrow-derived MSC (BMSC)-treated group normalized by day 14, while the weight in the control group was improved but not to pre-treatment levels. MSC, mesenchymal stem cell.

Following the third passage, the basic cell forms appeared to be relatively longer fusiform in shape, and were arranged uniformly (Fig. 1B).

Induced differentiation. At 5 days after the third passage, treated rat BMSCs were used as an osteogenic inducer, as a result of which the cellular structure gradually changed, with the enlargement of the cytoplasm, change in the shape of the cells from long spindle to polygonal or irregular, and the development of multiple pseudopodia. After 2 weeks of induction, $\sim 50 \%$ of the cells transformed into a polygonal spindle, 
A
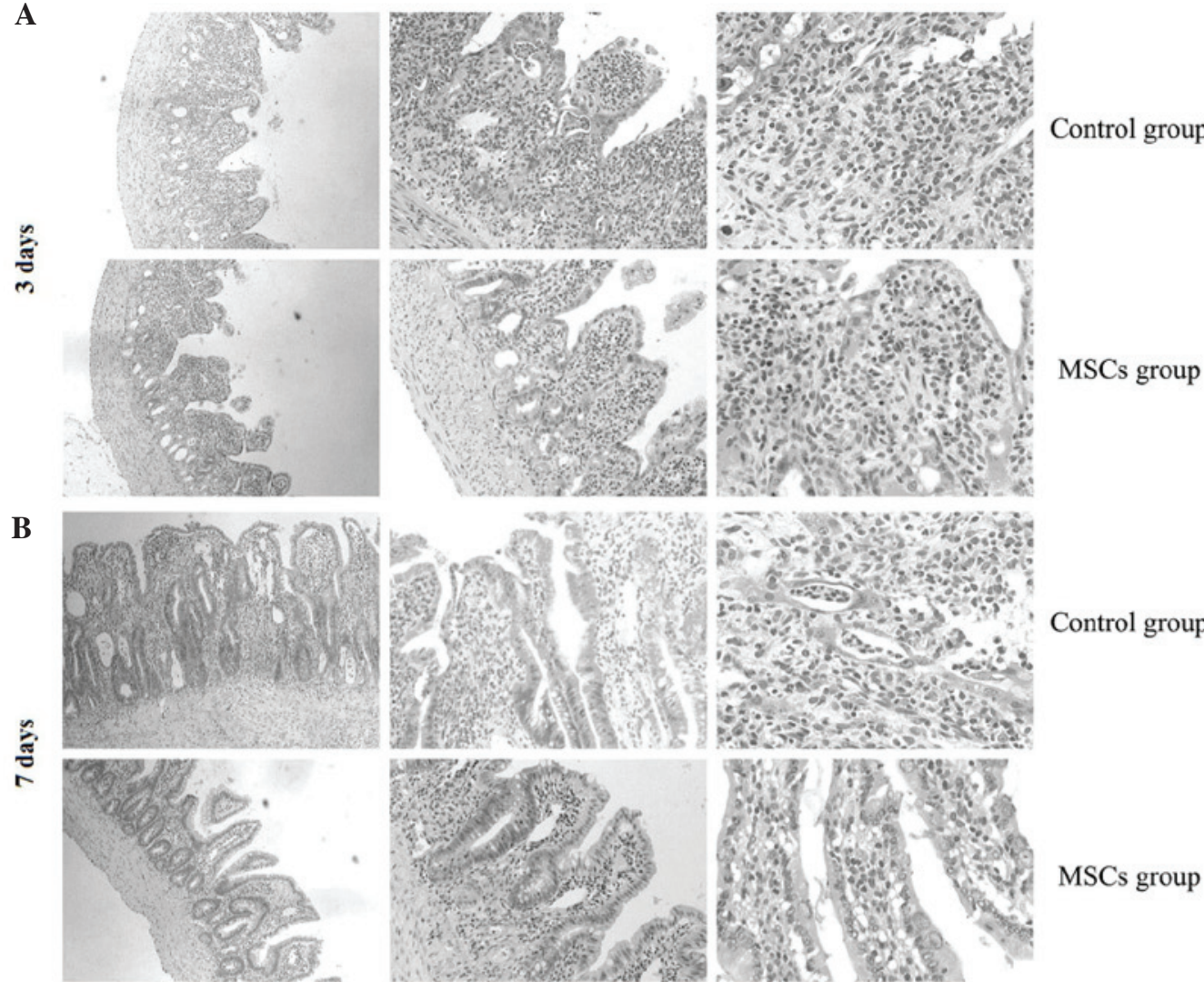

Control group
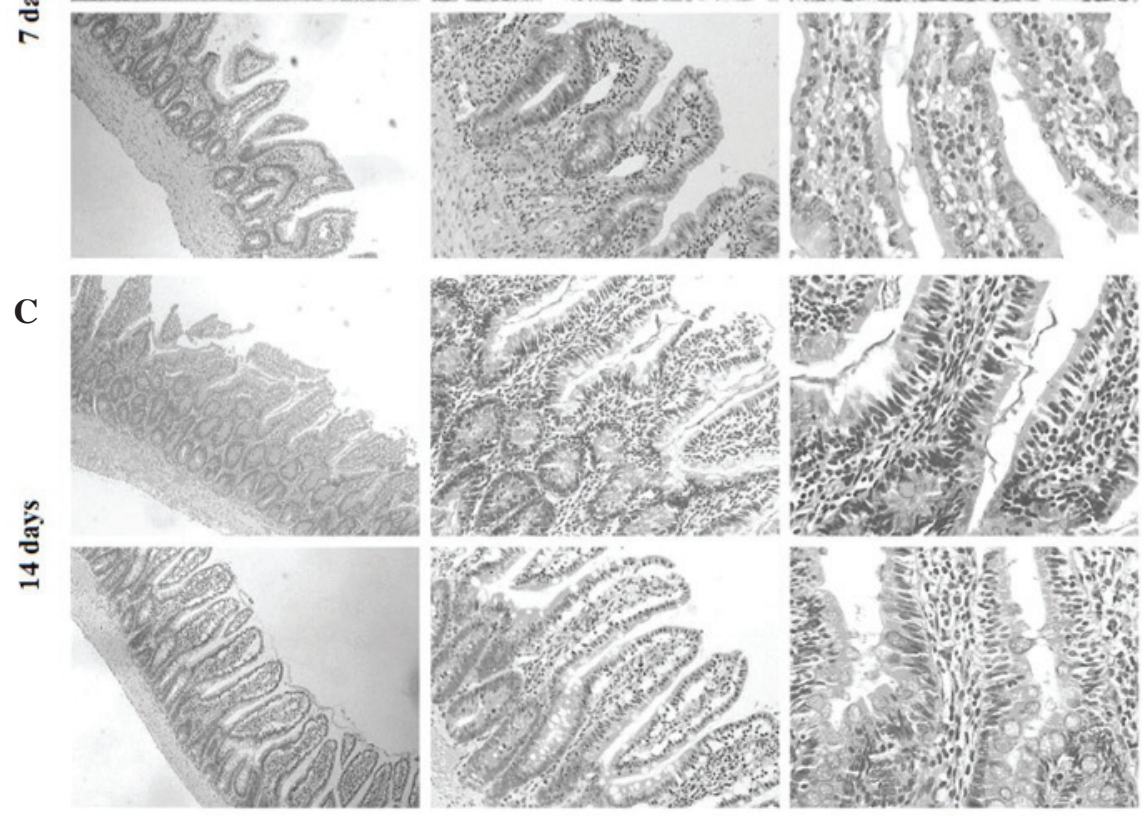

MSCs group

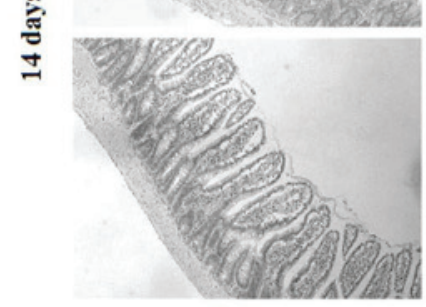

$250 \mu \mathrm{m}$

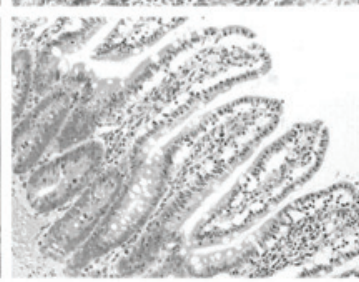

$100 \mu \mathrm{m}$

$50 \mu \mathrm{m}$

Figure 4. Morphological changes in the rat ileum. (A) On day 3 after irradiation, the structure of the rat ileum was disordered; a large number of intestinal epithelial cells were necrotic, 'pseudomembranous'-like structures formed, a large number of inflammatory cells infiltrated and the number of villi and glands significantly reduced. (B) On day 7 after irradiation, the structure of the rat ileum was still disordered; necrotic intestinal epithelial cells and pseudomembranous-like structures were evident. However, these conditions were significantly improved compared with those at day 3 . The number of villi and glands increased slightly in the BMSC-treated group. (C) On the day 14 after irradiation, the ileum villus tissues and gland hyperplasia were evident. The ileum structure in the bone marrow-derived MSC (BMSC)-treated group was clearly visible, hair growth was marked, aminae propria glands accumulated closely and were significantly improved compared with the control group. MSC, mesenchymal stem cell.

which grew in multiple layers and formed weak translucent cell nodules, with the initial characteristics of bone cells; the osteoblasts were slightly basophilic, with large and spherical nuclei and prominent Golgi apparatus that appear histologically as a clear zone adjacent to the nucleus. After 3 weeks, the number of nodules significantly increased. Von Kossa staining indicated that calcium nodules had been formed by bone mineralization (Fig. 1C).

At 3 days after third passage, the rat BMSCs were used as an adipogenic inducer, which resulted in the formation of small cytoplasmic lipid droplets in an irregular arrangement.
Between days 7 and 14, the number of cells containing lipid droplets gradually increased, the cellular morphology converted from the original long spindle to oval or irregular shape, and the number of fat droplets increased in number and fused gradually. Staining by Oil Red O revealed red-stained fat cells (Fig. 1D).

Surface antigen. Flow cytometry analysis detected the positive expression of the surface antigen CD29 on the cells. By contrast, CD90 cells of the cultured rat BMSCs did not express the antigens CD34 and CD45. The proportions of CD29+, 
Table I. Histological detection of ileal tissues following irradiation $(n=6)$.

\begin{tabular}{|c|c|c|c|c|}
\hline $\begin{array}{l}\text { Time after } \\
\text { irradiation }\end{array}$ & $\begin{array}{l}\text { Villus } \\
\text { height }(\mu \mathrm{m})\end{array}$ & $\begin{array}{c}\text { Intestinal } \\
\text { gland depth }(\mu \mathrm{m})\end{array}$ & $\begin{array}{l}\text { Radiation } \\
\text { injury score }\end{array}$ & $\begin{array}{c}\text { Plasma } \\
\text { Citrulline }(\mu \mathrm{g} / \mathrm{ml})\end{array}$ \\
\hline \multicolumn{5}{|l|}{ Day 3} \\
\hline Control & $211.46 \pm 11.52$ & $112.72 \pm 8.96$ & $15.50 \pm 1.68$ & $51.15 \pm 4.56$ \\
\hline BMSC-treated & $245.54 \pm 12.75^{\mathrm{a}}$ & $148.82 \pm 10.12^{\mathrm{a}}$ & $11.97 \pm 1.22^{\mathrm{a}}$ & $64.53 \pm 6.42^{\mathrm{a}}$ \\
\hline \multicolumn{5}{|l|}{ Day 7} \\
\hline Control & $296.30 \pm 16.65$ & $182.50 \pm 12.23$ & $10.65 \pm 1.37$ & $6.41 \pm 1.10$ \\
\hline BMSC-treated & $332.13 \pm 19.21^{\mathrm{a}}$ & $204.25 \pm 13.58^{\mathrm{a}}$ & $5.05 \pm 1.15^{\mathrm{a}}$ & $17.12 \pm 2.39^{\mathrm{a}}$ \\
\hline \multicolumn{5}{|l|}{ Day 14} \\
\hline Control & $339.14 \pm 19.21$ & $207.92 \pm 16.41$ & $8.26 \pm 1.03$ & $72.23 \pm 7.08$ \\
\hline BMSC-treated & $386.45 \pm 22.36^{\mathrm{a}}$ & $242.67 \pm 19.28^{a}$ & $3.68 \pm 0.45^{\mathrm{a}}$ & $91.99 \pm 9.87^{\mathrm{a}}$ \\
\hline
\end{tabular}

${ }^{\mathrm{a}} \mathrm{P}<0.01$ vs. control group. BMSC, bone marrow-derived mesenchymal stem cell.

$\mathrm{CD}^{+}{ }^{+}, \mathrm{CD} 4^{+}$and $\mathrm{CD} 45^{+}$cells were $99.25,98.37,1.12$ and $1.03 \%$, respectively (Fig. 2).

Rat weight and general condition. The body weight of the rats declined and reached the lowest point at day 7. The body weights of the BMSC-treated rats normalized by day 14 , whereas the weights of the control group rats were improved, but were not fully normalized to their pre-treatment weights. A significant differences was noted in body weight between the two groups $(\mathrm{P}<0.01)$ (Fig. 3). In addition, the rat mental state was poor following irradiation, with poor response to stimulation and reduced physical activity and food intake. After 2 days of irradiation, the rats in the control and BMSC-treated groups excreted thin feces containing mucus-like substance, indicating diarrhea. Two rats in the control group died on days 4 and 5 after irradiation and one rat in the BMSC-treated group died on day 5 after irradiation.

Morphology and radiation injury score. At day 3 after irradiation, the following observations were observed (Fig. 4): Disordered structure of the rat ileum, necrosis of a large number of epithelial cells to form necrotic villi with infiltration of a large numbers of inflammatory cells, and a significant reduction in the numbers of villi and glands. The ileum structure persisted, the numbers of mucosal epithelial necrosis cells and inflammatory cells were reduced, and the numbers of villi and glands were larger in the BMSC-treated group compared with the control group. At day 14 after irradiation, the ileum structure was clearly visible, the villi showed marked growth and the glands were formed more closely and were better structurally organized in the BMSC-treated group compared with the control group (Fig. 4).

The most severe effects of irradiation observed on day 3 included necrosis of the ileum glands and thinning of the mucosal layer. The intestinal tissues exhibited repair on day 7 after exposure. Furthermore, the height of the ileum villus and intestinal gland depth began to increase, whereas the radiation injury score decreased. The villus height and intestinal gland depth of the BMSC-treated group were significantly increased compared with the control group. The radiation injury scores were significantly reduced compared with the control group $(\mathrm{P}<0.01)$. At day 14 , the villus height and intestinal gland depth of the BMSC-treated group were similar to the normal intestinal tissue, and no significant improvement was observed on day 7 in comparison with the control group (Table I).

Plasma citrulline content. Following irradiation, the rat plasma citrulline content decreased significantly, reaching the lowest point on the day $7(\mathrm{P}<0.01)$. We speculated that the repair mechanism was initiated in the intestinal tract, as a result of which the plasma citrulline content began to increase. The recovery speed of the plasma citrulline content in the BMSC-treated group was significantly faster compared with the control group $(\mathrm{P}<0.01)$. The plasma citrulline content in the BMSC-treated group basically recovered to the near normal levels on day 14 (Table I).

Cytokine expression. IL-2 and PGE2 appeared to serve a crucial function in the inflammatory reaction. Under microscopic observation, IL-2 and PGE2 were primarily detected in the fibroblasts, inflammatory cells and intestinal epithelial cells. At day 3 after irradiation, the express levels of IL-2 and PGE2 in the control group were significantly increased compared with the BMSC-treated group $(\mathrm{P}<0.01)$.

$\mathrm{SDF}-1$ is one of the major chemokines in vivo. SDF-1 was predominantly expressed in the hair follicles around the wound margins, newborn glandular cells, fibroblasts and capillary endothelial cells. Following irradiation, the positive expression of SDF-1 in the BMSC-treated group appeared significantly increased compared with the control group $(\mathrm{P}<0.01)$ (Fig. 5).

\section{Discussion}

Intestinal tissues are highly sensitive to radiation and are among the most common sites of clinical radiation damage (11). Radiation-induced acute intestinal injury has been frequently reported in individuals exposed to nuclear accidents and radiation therapies for abdominal tumors, and there are currently no effective treatments for this damage (2). In the present study, a rat model of radiation-induced acute intestinal injury 
A

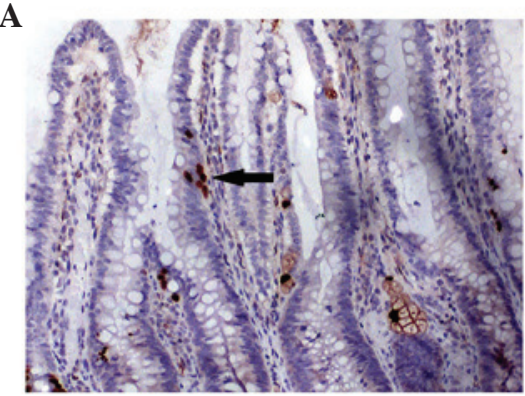

D
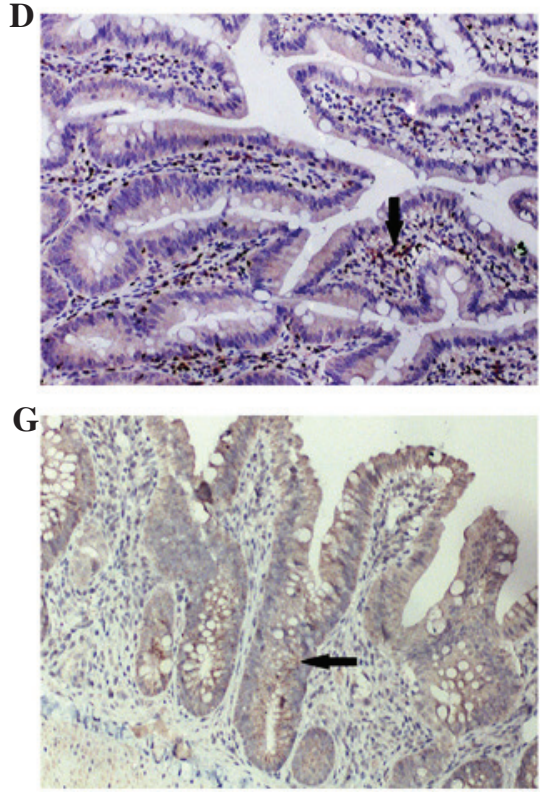

B
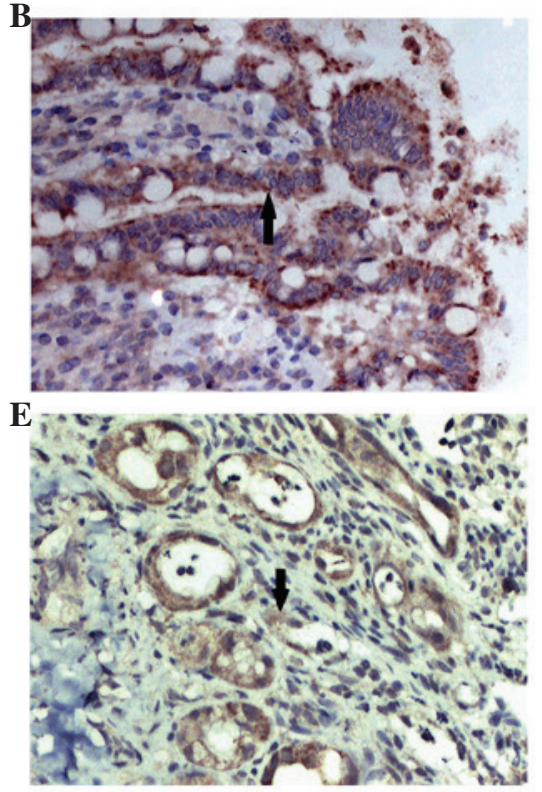

$\mathbf{H}$

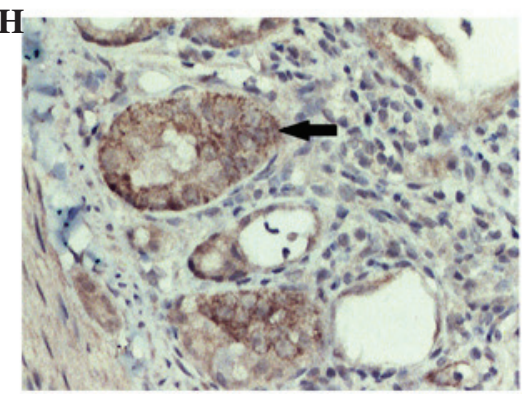

C
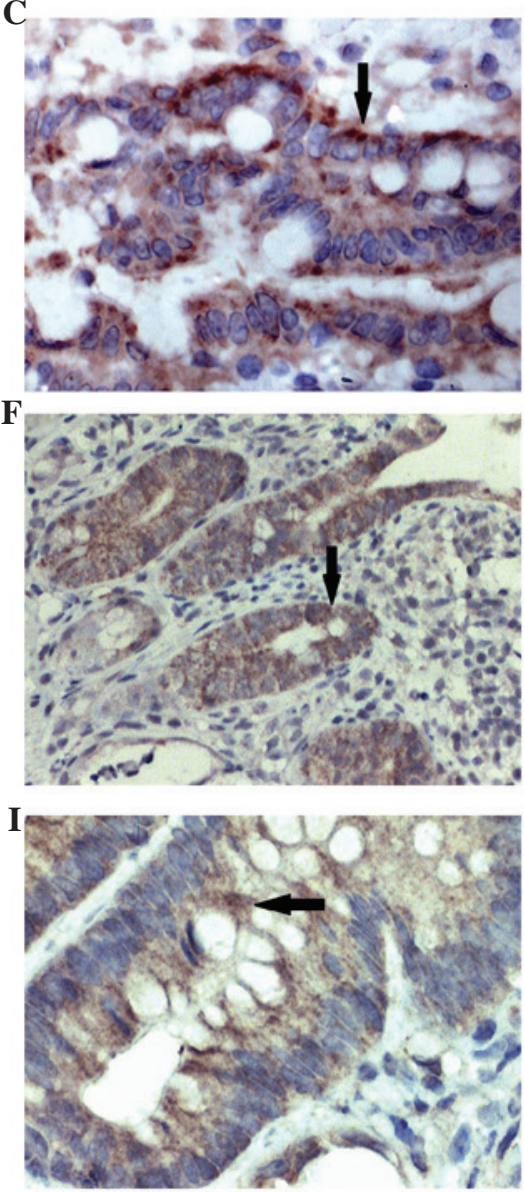

Figure 5. Detection results of cytokine expression in intestinal tissue. Interleukin-2 was predominantly distributed in the (A) intestinal epithelial cells (x40), (B) fibroblasts (x100) and (C) inflammatory cells (x200). Prostaglandin E2 was predominantly distributed in the (D) fibroblasts (x40), (E) capillary endothelial cells (x100) and (F) neonatal intestinal epithelial cells (x200). Stromal cell-derived factor 1 was predominantly distributed in (G) fibroblasts (x40), (H) inflammatory cells (x100) and (I) damaged intestinal epithelial cells (x200). Positive cells appeared as intracytoplasmic dark-colored particles.

was established, and the results indicated that BMSCs exert a protective effect in damaged intestinal tissues. BMSCs are able to promote the structural and functional repair of radiation-induced acute damage of intestinal tissues. Furthermore, BMSCs may inhibit inflammation and induce the secretion of cytokines to modify the local microenvironment in order to promote intestinal tissue reconstruction.

Chapel et al (12) used labeled MSCs to treat primates exposed to radiation and found that the labeled cells were undetectable in the damaged intestinal tissues following a number of months of transplantation. Linard et al (3) established a porcine model of colorectal radiation injury and performed multiple intravenous infusions of autologous BMSCs, which appeared to reduce local inflammatory cytokine expression and increase the expression of IL-10. The radiation-induced fibrosis was suppressed by reducing collagen deposition, transforming growth factor- $\beta$ expression and regulating the balance between matrix metalloproteinase and tissue inhibitors of metalloproteinases. In addition, Chang et al (4) injected human fat source MSCs (hAd-MSCs) into rats following complete irradiation of the rat stomach. The hAd-MSCs exerted a therapeutic effect, in addition to anti-inflammatory and pro-angiogenic effects. Gao et al (5) found that the intravenous injection of human umbilical cord-derived MSCs into BALB/C male mice following abdominal irradiation (10 Gy) improved the survival rate of rats and reduced the incidence of diarrhea.

In the present study, SD rats were exposed to a total dose of 12 Gy whole abdominal irradiation using a single linear medical accelerator. The results showed that the mental state of the rats was worsened following radiation exposure, with poor response to stimulation and reduced physical activity and food intake. Under microscopic observation, obvious mesenteric congestion, intestinal epithelial cell necrosis, destroyed glands, the formation of 'pseudomembranous'-like structure and a large number of inflammatory cell infiltration were visible. The intestinal villus height and the intestinal gland depth in the BMSC-treated cats were significantly increased compared with the control group after irradiation. The reduction in the intestinal radiation injury score indicated the protective effects of BMSCs against radiation injury.

Plasma citrulline is a functional parameter of intestinal epithelial cells, which indicates the total intestinal metabolism of the intestinal tract (13). Evaluation of plasma citrulline is simpler and more easily repeated compared with traditional detection methods for intestinal function, such as nitrogen balance (14), fecal fatty acid determination (15) and the D-xylose absorption test (16). The results of the present study showed that the recovery speed of the plasma citrulline in the BMSC-treated rats was significantly increased compared with 
the control group, suggesting that BMSCs are able to promote the recovery of intestinal function.

The aforementioned results suggest that MSC transplantation exerts a reparative effect in damaged organs, which may be achieved via the following mechanisms: MSCs differentiate into the target organ tissue type through horizontal differentiation or dedifferentiation, thus serving complementary and repair functions (17), and in addition, MSCs secrete various cytokines (18). The inflammatory response has been shown to be among the primary mechanisms underlying radiation damage (19). The results of the present study were consistent with these previous findings, as the expression levels of the inflammatory factors PGE2 and IL-2 in the intestinal tissues of the BMSC-treated group were significantly reduced compared with the control group. This result supported our hypothesis that MSCs are able to inhibit inflammation and regulate the local microenvironment in order to promote the repair of intestinal tissues damaged by radiation. SDF-1 and its receptor CXCR4 serve a crucial function in the stem cell-homing process (20). SDF-1 expression in the intestinal tissue of BMSC-treated tissues has been reported to be significantly increased (20). We hypothesized that BMSCs migration to the intestinal tract and their involvement in the repair of radiation-induced intestinal injury are closely associated with the SDF-1/CXCR4 axis. This may indicate the positive feedback mechanism of the BMSCs implanted intestinal tissues is mediated via the paracrine-stimulated secretion of SDF-1 by intestinal lamina propria stromal cells, which attracts increasing numbers of MSCs to accelerate the repair of intestinal tissues.

In summary, the present results suggest that BMSCs are able to inhibit the local inflammatory response, enhance the secretion of SDF-1 to promote the movement of BMSCs to radiation-damaged intestinal tissues and promote the repair of damaged intestinal structure and function. The application of MSCs in the treatment of radiation injury remains preliminary. The precise underlying mechanism of damage repair, and the optimum treatment dose and route remain unclear. Therefore, further studies are required to provide novel insights into the treatment of acute diseases caused by radiation exposure.

\section{Acknowledgements}

This study was supported by the Army 'Twelfth Five-Year' Science and Technology Key Project (grant no. BWS11J004), Nanjing Military Science and Technology Key Project (grant no. 10z031) and the Fujian Provincial Science and Technology Innovation Platform Project (grant no. 2010Y2006).

\section{References}

1. Akita S: Treatment of radiation injury. Adv Wound Care (New Rochelle) 3: 1-11, 2014.

2. Shadad AK, Sullivan FJ, Martin JD and Egan LJ: Gastrointestinal radiation injury: Prevention and treatment. World J Gastroenterol 19: 199-208, 2013.
3. Linard C, Busson E,Holler V, Strup-Perrot C,Lacave-Lapalun JV, Lhomme B, Prat M, Devauchelle P, Sabourin JC, Simon JM, et al: Repeated autologous bone marrow-derived mesenchymal stem cell injections improve radiation-induced proctitis in pigs. Stem Cells Transl Med 2: 916-927, 2013.

4. Chang P, Qu Y, Liu Y, Cui S, Zhu D, Wang H and Jin X: Multi-therapeutic effects of human adipose-derived mesenchymal stem cells on radiation-induced intestinal injury. Cell Death Dis 4: e685, 2013

5. Gao Z, Zhang Q, Han Y, Cheng X, Lu Y, Fan L and Wu Z: Mesenchymal stromal cell-conditioned medium prevents radiation-induced small intestine injury in mice. Cytotherapy 14: 267-273, 2012.

6. Francois S, Bensidhoum M, Mouiseddine M, Mazurier C, Allenet B, Semont A, Frick J, Saché A, Bouchet S, Thierry D, et al: Local irradiation not only induces homing of human mesenchymal stem cells at exposed sites but promotes their widespread engraftment to multiple organs: A study of their quantitative distribution after irradiation damage. Stem Cells 24: 1020-1029, 2006.

7. Okamoto R, Yajima T, Yamazaki M, Kanai T, Mukai M, Okamoto S, Ikeda Y, Hibi T, Inazawa J and Watanabe M: Damaged epithelia regenerated by bone marrow derived cells in the human gastrointestinal tract. Nat Med 8: 1011-1017, 2002

8. Herzog EL, Chai L and Krause DS: Plasticity of marrow-derived stem cells. Blood 102: 3483-3493, 2003.

9. Sémont A, François S, Mouiseddine M, François A, Saché A, Frick J, Thierry D and Chapel A: Mesenchymal stem cells increase self-renewal of small intestinal epithelium and accelerate structural recovery after radiation injury. Adv Exp Med Biol 585: 19-30, 2006.

10. Kawakatsu M, Urata Y, Goto S, Ono Y and Li TS: Placental extract protects bone marrow-derived stem/progenitor cells against radiation injury through anti-inflammatory activity. J Radiat Res 54: 268-276, 2013.

11. Kim JS, Yang M, Lee CG, Kim SD, Kim JK and Yang K: In vitro and in vivo protective effects of granulocyte colony-stimulating factor against radiation-induced intestinal injury. Arch Pharm Res 36: 1252-1261, 2013.

12. Chapel A, Bertho JM, Bensidhoum M, Fouillard L, Young RG, Frick J, Demarquay C, Cuvelier F, Mathieu E, Trompier F, et al: Mesenchymal stem cells home to injured tissues when co-infused with hematopoietic cells to treat a radiation-induced multi-organ failure syndrome. J Gene Med 5: 1028-1038, 2003.

13. Lutgens L and Lambin P: Biomarkers for radiation-induced small bowel epithelial damage: An emerging role for plasma Citrulline. World J Gastroenterol 13: 3033-3042, 2007.

14. Boutry C, Matsumoto H, Bos C, Moinard C, Cynober L, Yin Y, Tomé D and Blachier F: Decreased glutamate, glutamine and citrulline concentrations in plasma and muscle in endotoxemia cannot be reversed by glutamate or glutamine supplementation: A primary intestinal defect? Amino Acids 43: 1485-1498, 2012.

15. Walton C, Fowler DP, Turner C, Jia W, Whitehead RN, Griffiths L, Dawson C, Waring RH, Ramsden DB, Cole JA, et al: Analysis of volatile organic compounds of bacterial origin in chronic gastrointestinal diseases. Inflamm Bowel Dis 19: 2069-2078, 2013.

16. Spallek A, Recknagel S, Breuer J, Koeller G and Schusser GF: Influence of laxatives on gastric emptying in healthy Warmblood horses evaluated with the D-xylose absorption test. Berl Munch Tierarztl Wochenschr 126: 245-250, 2013.

17. Das M, Sundell IB and Koka PS: Adult mesenchymal stem cells and their potency in the cell-based therapy. J Stem Cells 8: 1-16, 2013.

18. Fernández Vallone VB, Romaniuk MA, Choi H, Labovsky V, Otaegui $\mathrm{J}$ and Chasseing NA: Mesenchymal stem cells and their use in therapy: What has been achieved? Differentiation 85: 1-10, 2013.

19. Anuranjani and Bala M: Concerted action of Nrf2-ARE pathway, MRN complex, HMGB1 and inflammatory cytokines-implication in modification of radiation damage. Redox Biol 2: 832-846, 2014.

20. Marquez-Curtis LA and Janowska-Wieczorek A: Enhancing the migration ability of mesenchymal stromal cells by targeting the SDF-1/CXCR4 axis. Biomed Res Int 2013: 561098, 2013. 